\title{
Effects of Extremity Elevation and Health Factors On Soft Tissue Electrical Conductivity
}

\author{
J. R. Feldkamp and J. Heller \\ Kimberly-Clark Corporation, Corporate Research and Engineering \\ 2100 Winchester Road, Neenah, WI 54956, U.S.A
}

\begin{abstract}
Two clinical studies were completed using an auto-tuned induction coil conductivity sensor (ICCS) to determine the effects of a variety of factors on the electrical conductivity of soft tissue. In addition to fifteen "subject variables" such as blood pressure and others, we have specifically focused on considering the role of such factors as gender, age, BMI, smoking and elevation of extremities. Measurements were made at seven sites on either side of the body for a total of fourteen. Higher conductivities were obtained for women than men at all sites. At five sites, where age was a significant factor, conductivity was found to decline with increased age. Interestingly, smokers as a group tended to have reduced conductivity, suggesting that aging and smoking have similar effects on the microvasculature of soft tissue. Generally speaking, electrical conductivity is observed to increase in response to increased elevation at sites located on extremities. Considering just healthy adults, a definite pattern of elevation-induced electrical conductivity displacement emerges when subjects are flagged according to high, low or moderate blood pressure. We suggest that violations of this pattern may provide a method for identifying those individuals in an early stage of peripheral vascular disease.
\end{abstract}

Keywords: Induction coil, electrical conductivity, soft tissue, extremity elevation, vascular disease

\section{INTRODUCTION}

$I_{t}^{\wedge}$ A PREVIOUS PAPER [1], the authors described autotuned induction coil technology which was shown to be an effective tool for measurement of in-vivo electrical conductivity. Using phase-locked-loop (PLL) circuitry, measurement at a fixed excitation frequency was assured, in spite of changes in coil capacitance resulting from coil-body contact. As stated there, several of the disadvantages associated with electrode methods for electrical conductivity measurement are avoided since our method does not require electrical contact with the body.

This paper reports on the use of the induction coil conductivity sensor (ICCS) in two different clinical trials, the first focusing on establishing normal conductivity patterns in healthy human subjects. The second study aims to ascertain whether ICCS can detect departures from normal electrical conductivity patterns in subjects that are likely to exhibit signs of compromised health, due to age, obesity, smoking or other reported conditions. A motivation for these studies partly comes from a wide variety of literature results, ranging from electrical impedance tomography [2] to conductivity measurements on specific tissues [3]. In either case, the desire is to develop a strategy that allows detection of abnormalities.

A particular discovery described in our earlier report, that we hoped to exploit in our clinical trials is that electrical conductivity in an extremity typically increases when elevated above the heart. Particular goals are to understand what factors may contribute to elevation-induced conductivity changes and how compromised health might cause departures from "normalcy". In order to provide a framework for our interpretation of electrical conductivity values, we have proposed using a simple human tissue model - a porous medium consisting of two phases. One of these phases consists of a relatively non-conducting microvasculature [4] that includes arterioles, capillaries, venules and lymph vessels, while the second phase consists of highly conductive interstitial fluids. Redistribution of fluids between the two phases, especially due to elevation changes in extremities, is expected to cause measurable changes in electrical conductivity. In addition to the effects of elevation changes, we also seek to understand how electrical conductivity may depend on a variety of "subject variables", such as age, weight, height, etc. An expectation of electrical conductivity dependency on such common subject variables is supported by Jayasree et. al. [5], where age was shown to have an effect on photo-plethysmography (PPG) systolic peaks, which provide a measure of blood volume. There, the area of PPG systolic peaks obtained from the right index finger was shown to increase with age, not because blood vessels expand more so in older adults compared to younger counterparts, but rather because aged vessels fail to relax (see their figure 1). Given their results, we were encouraged to explore a possible age-dependency for electrical conductivity.

\section{CLINICAL DESIGN - PART I}

As indicated in our previous work [1], the ICCS instrument targets soft tissues residing in a region extending from the skin surface to a depth of about $15 \mathrm{~mm}$ beneath the epidermal surface. In either clinical, we identified seven locations on each side of the body as measurement sites, for a total of fourteen. Sites included: 1) mid-volar forearm (M); 2) proximal volar forearm (P); 3) inside upper $\operatorname{arm}(\mathrm{U}) ; 4)$ lumbosacral (S); 5) inside mid-thigh (T); 6) back of calf (C); and, 7) bottom of foot (F). Electrical conductivity at each site was measured in triplicate, with each of the 14 sites visited sequentially before another replicate was obtained.

For clinical I, each subject was asked to visit the clinic a total of four times for a complete set of measurements. At the first visit to the clinic, marks were made with a surgical pen to facilitate return to the same location at later visits, as well as 
from one replicate to the next. The first two sets of measurements, taken four hours apart, preceded the third and fourth sets, also spaced four hours apart, by two weeks.

During measurement, subjects were able to sit comfortably on an examination table, with torso upright and legs extended at hip elevation. Prior to collecting a set of measurements, subjects were given 15 minutes to relax and acclimate to surroundings - temperature in the exam room was held at $72 \mathrm{~F}$ while humidity was kept about $40 \%$. Forty healthy subjects were recruited which included 16 men and 24 women. Subjects were distributed uniformly over the age range of 25 to 45 . Subsequent to collection of data at the chosen sites, a final measurement, one replicate only, was taken at the midvolar forearm of the right arm after it was kept elevated above the head for 60 seconds.

Subjects were judged healthy provided that they were nonsmokers, had BMI less than or equal to 27 and not routinely on medication for treatment of cardiovascular issues. In addition to ICCS measurements, we also measured: weight, height, blood pressure (both arms), urine specific gravity, pulse, BMI, percent body water, percent body fat and bone mass. The latter three parameters were measured using a Tanita scale (model BC-534). Blood pressures were measured in both arms because of a reported correlation between vascular disease and blood pressure disparities [6].

Throughout our first clinical study, two nurses were employed to maintain higher throughput. While a nurse positioned an ICCS unit on a particular site, the authors acquired data on a nearby laptop computer via Bluetooth. Upon vocal communication from the nurse that the ICCS unit was in position, a measurement was acquired with a mouse click. Very early on, some conductivity data were observed to be much lower than expected. This was traced to a nurse's tendency to move away from a site before being instructed to do so. Apparently the "mouse click" was audible and misinterpreted to indicate measurement completion. Each measurement required that the instrument remain at a site for about a second, so that 40 samples could be acquired, averaged and returned via Bluetooth. After observing the low values, further clarification and implementation of proper measurement protocol eliminated the issue. However, as a result, seven sets of measurements (out of 160) were removed from statistical analyses in the first clinical study.

\section{Clinical Results - PART I}

Of particular interest is the establishment of normal patterns of electrical conductivity values in healthy adults. One observation, made even during the course of collecting data, was that women exhibit higher conductivity than men. Upon further analysis, differences were found to be more substantial at some body sites than others. Overall, electrical conductivity is about $0.35 \mathrm{~S} / \mathrm{m}$ higher in women than in men. Differences by site are shown in the paired comparison test of figure 1. Obviously, the smallest differences are found in the feet (F) and lumbosacral regions (S), while the greatest differences are found in the thighs (T) and calf (C). Particularly reassuring is that data associated with right and left side body sites tend to group together as they should - i.e., right calf with left calf, and so on. This is the pattern expected for a sampling of healthy adults.

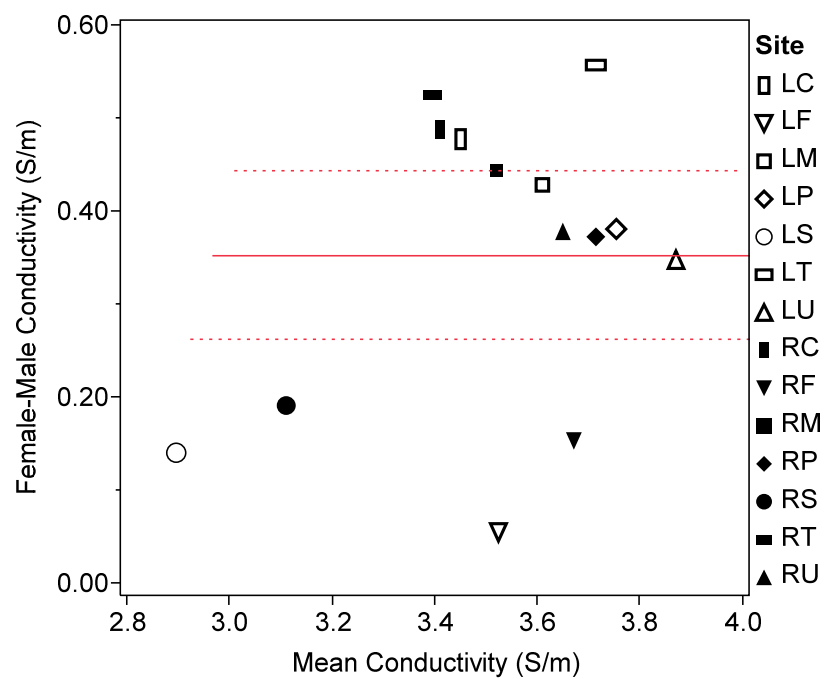

Fig.1 Paired comparison of average male and female electrical conductivities by site. Solid symbols are used for right-side locations and open for left side (t-ratio $=8.43$ ).

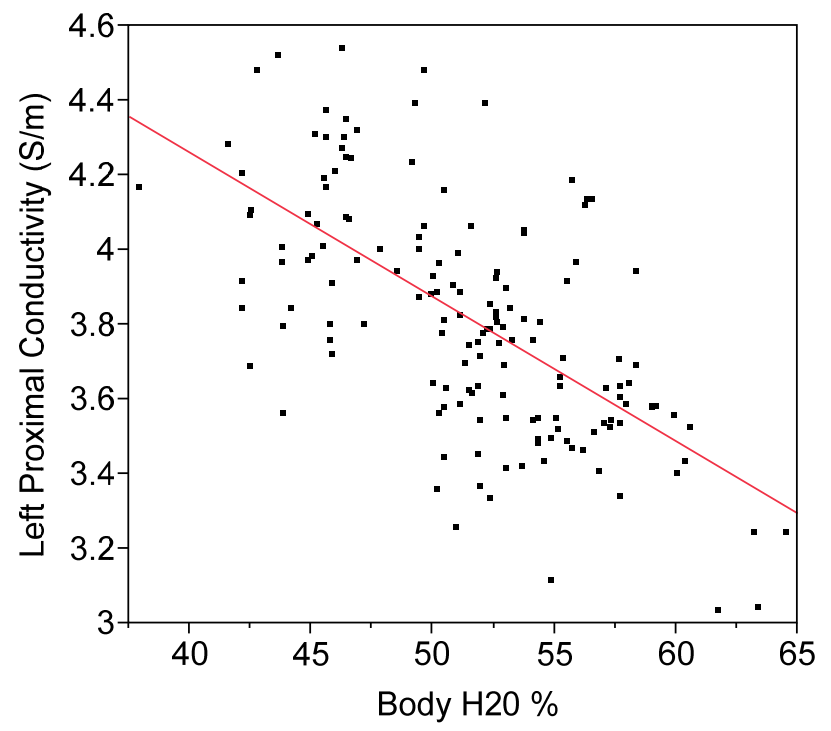

Fig.2 Conductivity at the left proximal volar forearm as a function of percent body water $(\mathrm{R}$-squared $=0.41)$.

In an effort to understand what variables might explain the observed gender differences, a scatter plot matrix was created in JMP (8.0.1; SAS Inst. Inc.). Very quickly, a correlation was "observed" between gender and percent body water (Tanita scale) as well as a correlation between percent body water and electrical conductivity. Percent body water averaged about $55.0 \pm 3.54$ for men and about $49.08 \pm 4.66$ 
for women. It is very tempting to attribute the $10 \%$ difference in conductivity to the $10 \%$ difference in body water. In order to solidify the connections between percent body water, electrical conductivity and gender, electrical conductivity would need to decrease as percent body water increases. Such a connection was found at nearly every site - one example is given in figure 2 for the left proximal volar forearm.

Further effort was made to understand the dependency of electrical conductivity on our measured subject variables by building a partial factorial statistical model that included all main effects and two-way interactions between main effects. Because of the high correlation among some of our subject variables, not all were included in the statistical model. For example, body water and fat show high inverse correlation (Rsquared $=0.94)$, so body fat was dropped. Because of the connection between gender and body water, gender was not used. A stepwise fit model was built whereby terms were added provided the significance probability fell beneath 0.05 and were removed if above 0.05. Except for the feet where correlation was poor (R-squared $<0.25$ ), R-squared values for built models ranged from about 0.60 to 0.75 .

Based upon ranking of t-ratios and number of body sites where a variable was shown to have a significant connection to conductivity (|t-ratio| > 2.0), variables having the most important influence on conductivity were percent body water, weight, BMI, height, blood pressure and age. Those with little or no connection included bone mass, urine specific gravity and pulse. Because of the relatively high linear correlation between blood pressures (R-squared $\sim 0.7$ ), it was not possible to determine if one of the four pressures most influenced electrical conductivity. For most sites, increased blood pressure was observed to lead to somewhat increased conductivity. In those cases where conductivity showed age dependency (5 sites), conductivity always declined with increased age.

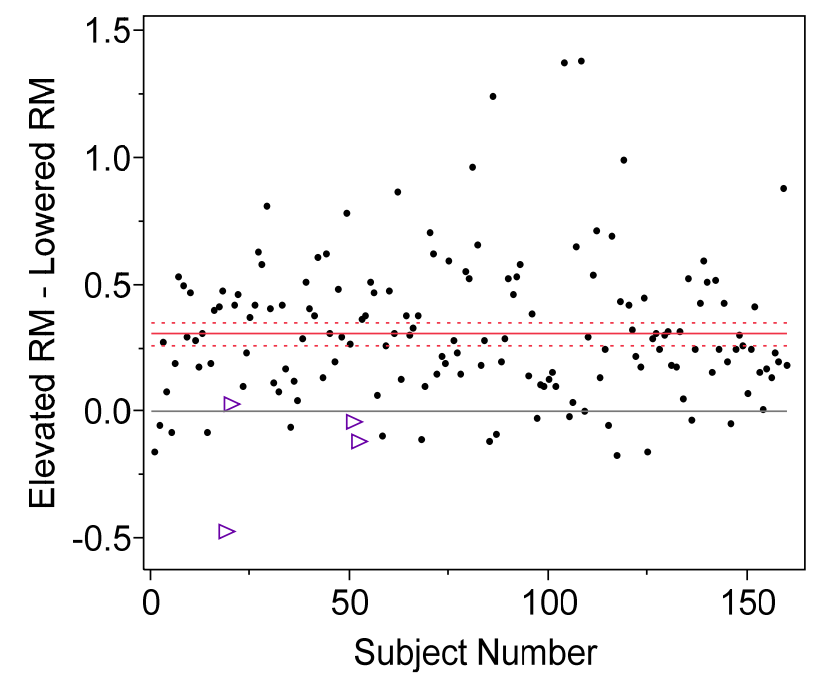

Fig.3 Effect of elevation of right mid-volar forearm on electrical conductivity. All four of the data points belonging to the outlier (same subject) are shown with triangular symbols.
As discussed briefly in our companion paper [1], increased extremity elevation was shown to lead to increased electrical conductivity. Here we explored that effect with just the right mid-volar forearm. Figure 3 plots the conductivity displacement in response to elevating the mid-volar forearm for a period of 60 seconds prior to measurement. One subject showed a negative displacement that appears to be significant, so all four results from that subject are shown with open triangles. Upon further investigation, these data points, as well as others that showed little or no conductivity displacement, appeared to be associated with elevated systolic blood pressure. To consider the effect further, figure 4 plots conductivity displacement once again, but this time for male subjects only, and with upward directed triangles used in those cases when systolic blood pressure in either arm was greater than or equal to $120 \mathrm{~mm} \mathrm{Hg}$ and downward directed triangles when diastolic blood pressure in either arm is less than or equal to $65 \mathrm{~mm} \mathrm{Hg}$. Only male subject data were used in figure 4 to remove the effect of gender, and because of a desire to provide a benchmark for stage II clinical work where only male subjects were included.

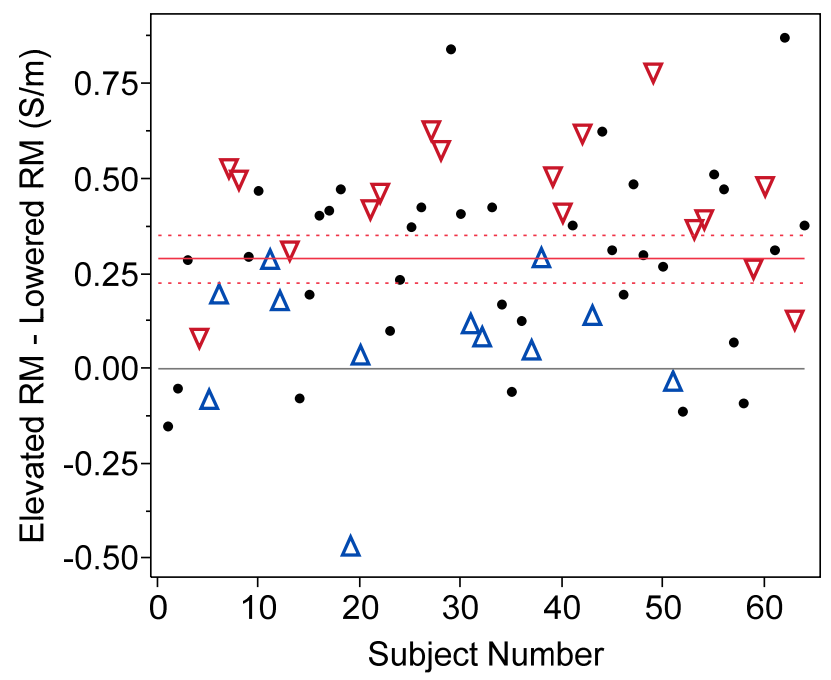

Fig.4 Conductivity shift at right mid-volar forearm in response to 60s of elevation - male subjects only. Upward triangles: SBP $>=$ 120; downward triangles: DBP $=<65$.

Clearly, elevated systolic blood pressure is implicated as a key factor influencing the extent to which electrical conductivity rises (or does not rise) in response to elevation change. Note that not a single high blood pressure subject contributes to the pool of data located above the mean conductivity displacement. It is also clear that low diastolic blood pressure contributes to a more dramatic upward response to elevation change. Note that no subject identified as having low diastolic BP in the right arm exhibits a negative conductivity shift - only three data points fall beneath the mean conductivity shift.

Nevertheless, low or high blood pressure alone cannot be the only factors influencing elevation-related conductivity 
shifts. In view of the data scatter, other variables such as extremity temperature (not measured) and height are also likely to play a role. The latter parameter would affect the amount of elevation change possible for subjects as well as any characteristic relaxation time for drainage - taller individuals may require more time to "drain" their microvasculature.

\section{CLINICAL DESIGN - PART II}

Clinical work described in the last section was meant to provide a kind of benchmark, to which an arbitrary subject could be compared. As such, only healthy individuals were considered. Here we describe a second clinical study that includes a broader range of subjects, in particular those whose health is likely compromised in some way. This clinical study was restricted to male subjects, primarily because of the known higher incidence of peripheral vascular disease (PVD) in males, but also because of a desire to avoid the effects of menstruation or menopause.

In this second study, 39 male subjects were divided into six risk groups, with group ' 1 ' judged to be most healthy and group six least healthy. Ranking was based upon just three risk factors: age, BMI and smoking. Smoking was quantified according to whether a subject smoked more than two cigarettes per day, or had not been smoking at all for at least 10 years - i.e., there was no attempt to introduce a smoking variable that actually reflected the number of cigarettes used per day. Table 1 shows the distribution of subjects over the six risk groups and how they were ranked according to risk.

\begin{tabular}{|c|c|c|c|}
\hline DOE Grid & \multicolumn{2}{|c|}{ Non Smoker } & Smoker \\
\hline Age Range: & $25--35$ & $36--70$ & $40--70$ \\
\hline $\mathrm{BMI}<28$ & $7(1)$ & $5 \quad(2)$ & $7(5)$ \\
\hline $\mathrm{BMI}>=28$ & $5 \quad(3)$ & $7 \quad(4)$ & $8(6)$ \\
\hline
\end{tabular}

Table 1: Distribution of 39 male subjects in second phase ICCS Clinical Study - risk level is shown in parentheses.

Numbers in parentheses designate both the group number and risk level assigned to that group. Due to its known connection with many health issues, high BMI was considered a greater risk factor than age in assigning groups two and three. Furthermore, many of the subjects indicated previous weight changes (loss or gain) and other health changes during interview, so that at some time in the past, a particular subject might not have qualified for the category in which they were placed for this study. Age and BMI distributions are shown in figure 5. BMI breakdown for risk group 1 was: 21.5, 21.6, 25.1, 25.5, 26.1, 27.1 and 27.4.

Through further evaluation of individuals, additional risk factors were identified. These included high blood pressure; asymmetric blood pressure; and, diabetes. Generally, a subject was viewed as having high blood pressure if systolic blood pressure in both arms and during both visits exceeded $120 \mathrm{~mm} \mathrm{Hg}$. A subject was considered to have an asymmetry in blood pressure if the difference in average systolic pressure between left and right arms exceeded $5 \mathrm{~mm} \mathrm{Hg}$. Studies reported in the literature [6] have shown PVD to be significantly more prevalent in those with blood pressure disparity. A subject was considered to have diabetes if that information was volunteered - no effort was made to verify a diabetic condition. Four subjects claimed to have diabetes, all of whom fell into the smokers group.

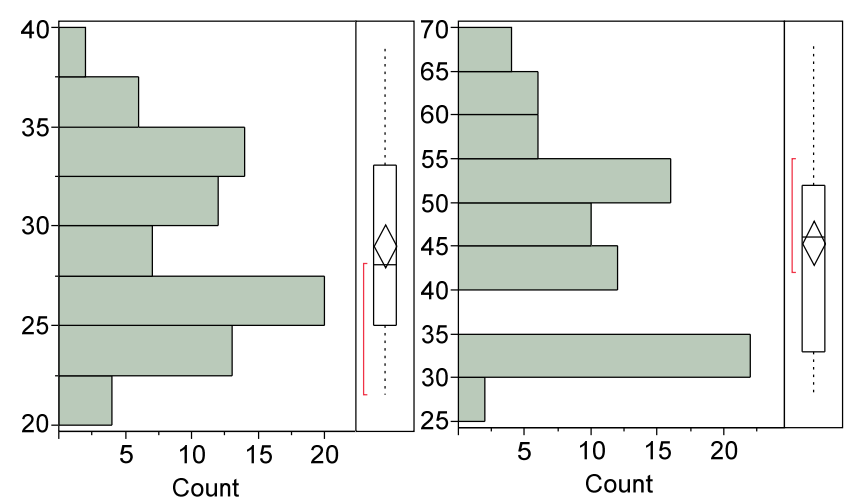

Fig.5 BMI (left) and age (right) distributions for the all-male study group. Note that each subject contributes twice, once for each of the two visits to the clinic.

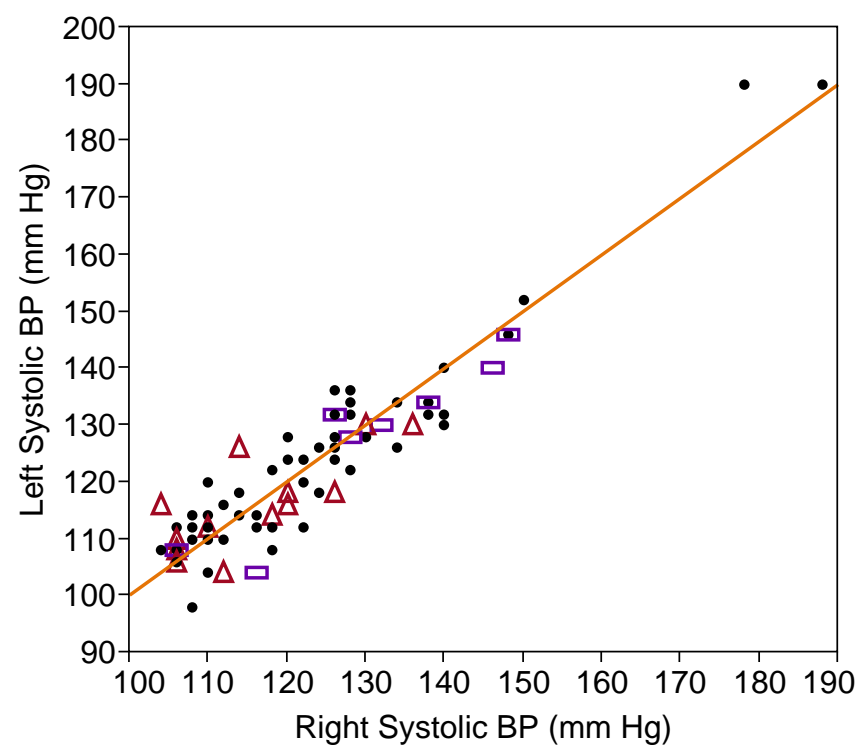

Fig.6 Comparison of systolic blood pressures $($ triangles = group 1; rectangles $=$ diabetics). Straight line has zero intercept and unity slope to help visualize BP disparity. 
In order to provide an indication of blood pressure distribution and also the extent to which blood pressures are correlated, figure 6 plots left against right systolic blood pressure, twice per subject for each of their two visits. Linear correlation of systolic pressures is good (R-squared 0.87). The line shown, though, is not the best fit line but rather a straight line with zero intercept and unit slope. Deviation of any point from the line is indicative of blood pressure asymmetry for that particular visit - each subject visited the clinic twice during their assigned day.

In addition to electrical conductivity measurements, the same set of subject variables as before were recorded for each of the two visits per subject. The only new variable is the non-continuous smoking variable. Our 14 subject variables, as previously noted, are not all independent. Using principle component analysis (PCA), only about six subject variables are needed to capture subject variability contained in the set.

Electrical conductivity measurements were made in triplicate at the same sites as before and in the same manner. One exception to the procedure was that measurements on the forearm were taken with forearms hanging at the side rather than held straight out as before. Once all regular replicates were obtained, measurements on elevated extremities were made in triplicate, with all replicates obtained at the same time. These included: a) raised proximal volar forearms, both left and right while in the sitting position; b) raised calves, both left and right with the subject lying flat on his back; c) calves while in the standing position. For each of the elevated positions, or for the standing position, the subject remained in that position for 30 seconds prior to ICCS measurement. While in the standing position, subjects were asked to distribute their weight equally on both feet. When measurement was made on one leg, the subject was asked to put their weight entirely on the other leg to relax the calf muscle being measured. Throughout the two weeks of the clinical, room temperature was maintained about $68 \mathrm{~F}$. Obvious changes in this clinical study are the addition of new elevated sites, elevated proximal volar rather than mid forearm, 30 second wait-time rather than 60 seconds, and a cooler temperature, by $4 \mathrm{~F}$.

\section{Clinical Results - Part II}

Overall, mean values of conductivity for body sites (not elevated) were not significantly different between the two clinical data sets for men, with the exception of the feet. Differences in average electrical conductivities between the two studies are given by location in table 2 (second clinical mean minus first clinical mean). In all cases except for the feet, standard deviations are larger than the measured mean differences - values for the feet are shaded. Higher than expected conductivities for the feet were noted during the study and considered puzzling until the attending nurse mentioned that most subject's feet felt cold to the touch. Given that the room was $4 \mathrm{~F}$ cooler for the second clinical trial, the elevated electrical conductivity was judged to result from reduction of blood flow to the feet in response to lowered ambient temperature. Correspondingly, the volume fraction allocated to the "insulating" microvasculature is reduced, with increased electrical conductivity expected.

\begin{tabular}{|l|c|c|c|c|}
\hline & \multicolumn{4}{|c|}{ Mean Conductivity Differences (S/m) } \\
\hline & L & L-std & $R$ & R-std \\
\hline $\mathrm{M} \mathrm{(arm)}$ & 0.17 & 0.295 & 0.25 & 0.265 \\
\hline $\mathrm{P}$ (arm) & 0.11 & 0.32 & 0.19 & 0.26 \\
\hline $\mathrm{U}$ (arm) & -0.1 & 0.32 & 0.2 & 0.28 \\
\hline $\mathrm{S}$ (back) & -0.04 & 0.19 & -0.17 & 0.255 \\
\hline $\mathrm{T}$ (thigh) & -0.13 & 0.35 & 0.01 & 0.335 \\
\hline $\mathrm{C}$ (calf) & 0.02 & 0.265 & 0.11 & 0.23 \\
\hline $\mathrm{F}$ (foot) & 1.16 & 0.37 & 0.84 & 0.295 \\
\hline Overall: & 0.17 & 0.30 & 0.20 & 0.27 \\
\hline
\end{tabular}

Table 2: Mean value differences in electrical conductivity between first and second clinical trials, by location. L-std and R-std refer to average standard deviations for two studies.

Aside from the feet, mean conductivities are the same as before, so "abnormal" conductivity values would have to be identified by comparison of subsets within the sample to other subsets. An especially effective strategy for accomplishing this is to build model(s) relating a subset of the conductivity data to subject variables, and then overlaying another "select" subset onto the model prediction curve. If one subsample is no different than another, then the two sets of data should overlay each other. This is precisely what has been done in our attempt to distinguish the impact of cigarette smoking on electrical conductivity.

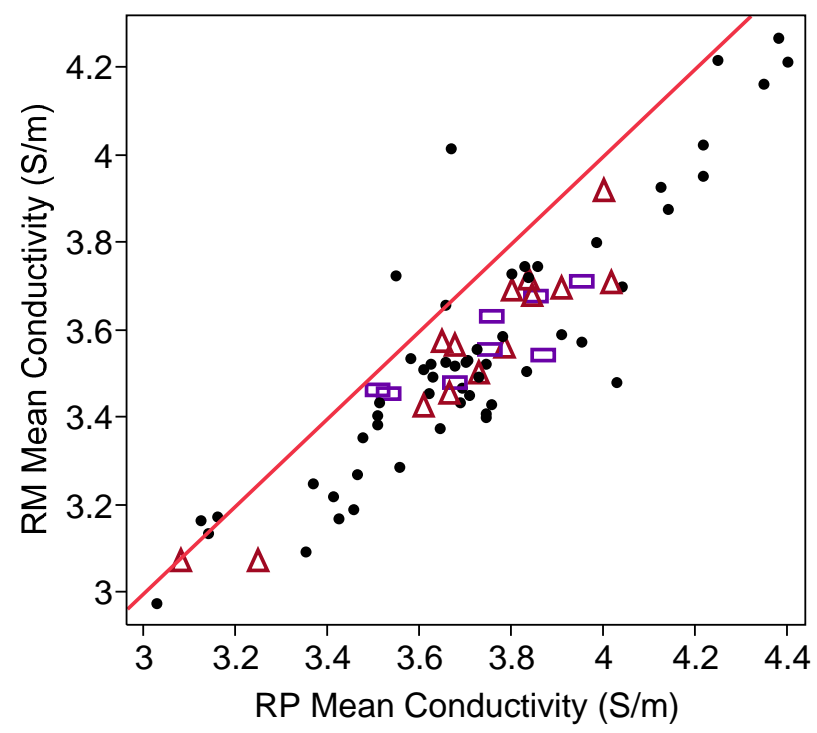

Fig.7 Plot comparing conductivities of right mid volar forearm to right proximal volar forearm (triangles = group 1; rectangles = diabetics). Zero intercept, unit slope line is shown to aid comparison. 
Assuming that smoking ought to contribute to increased PVD risk, model building focused on extremities, in particular the upper extremities. Three sites were probed by ICCS on each arm. But, performing a PCA on the arm data showed that all of these are highly correlated with each other. Thus, the effects of smoking on electrical conductivity, if present, should be apparent on an overlay plot built from any of the locations.

As an example of site-to-site correlation of electrical conductivities, figure 7 plots the proximal volar versus mid volar electrical conductivity on the right forearm. Clearly, conductivity at the two locations is well correlated. However, we note that most values fall beneath a line of zero intercept and unit slope, indicating generally lower conductivities at more distal forearm locations. In hindsight, this suggests that our change in location from mid to proximal volar forearm for study of elevation change effects on conductivity can be expected to result in smaller electrical conductivity displacements. As noted earlier, conductivity of the forearms in their lowered position was measured while arms were left free to hang at a subject's side. A plot similar to figure 7 was obtained for the left forearm, but with less difference between mid and proximal locations.

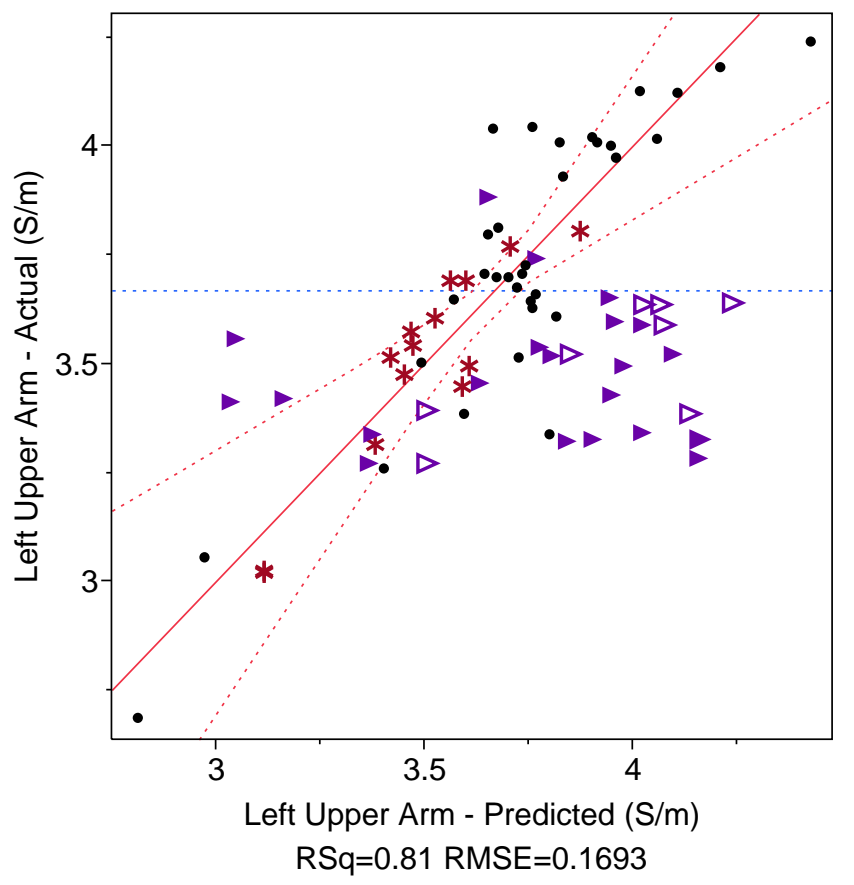

Fig.8 Overlay of smoker conductivities (triangles) onto plot of conductivity model for inside left upper arms of nonsmokers. Open triangles belong to diabetics, starred points belong to risk group one and remaining data belong to nonsmokers not in risk group one.

Figure 8 shows the result of building a model that attempts to relate electrical conductivity at the inside left upper arm to subject variables, but with smokers excluded.
The strategy for building a fit model was described in a previous section. Important main effects (|t-ratio|>2) were percent body water, BMI, weight and height - in that order. As before, decreased electrical conductivity follows from increased percent body water.

Using triangle symbols, conductivities for smokers are overlaid onto the fit model in figure 8. Diabetics, all of whom were smokers, are shown by the open triangle symbols. Clearly, data for smokers departs significantly from the trend followed by nonsmokers - only three smoker data points fall inside the 95\% confidence interval, and only two smoker data points exceed the mean conductivity. Furthermore, most smoker data points reside well to the right of the 95\% confidence interval curve, indicating that smoker conductivity should have been much higher than what was actually observed. We note here that smaller than expected conductivity is essentially the same effect already observed from the first clinical trial in connection with aging. This strongly suggests that smoking has the same effect as aging.

Just to convince ourselves that figure 8 is "reproducible", a similar plot was constructed for data collected at the inside upper right arm - shown in figure 9. Building a fit model for nonsmokers produced main effects (|t-ratio|>2) percent body water, age, BMI and height. As before, t-ratio is negative for an age effect.

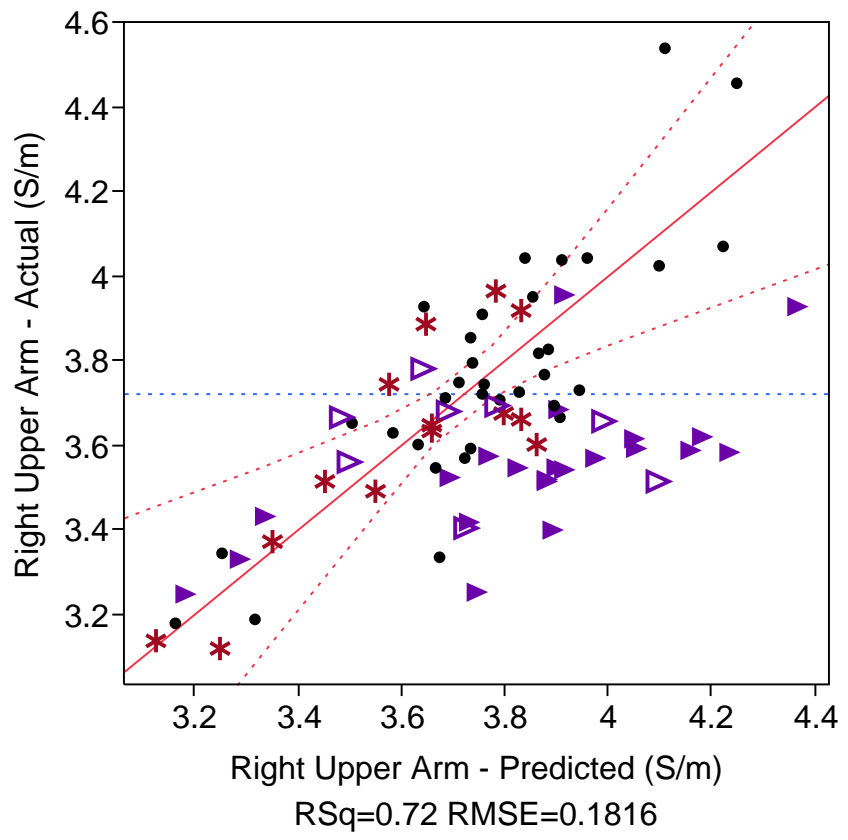

Fig.9 Overlay of smoker conductivities (triangles) onto plot of conductivity model for inside right upper arms of nonsmokers. Open triangles belong to diabetics, starred points belong to risk group one and remaining data belong to nonsmokers not in risk group one.

Though not as striking as was observed with the left arm, figure 9 still reveals a strong tendency for smoker 
conductivities to be too small. Thus, smoking appears to produce the same effect as aging on measured electrical conductivity of soft tissue when considering upper extremities. The contrast between smokers and nonsmokers was found to be very strong once again for the right calf, but without showing the strong trend toward lower than expected conductivity. As figure 10 shows, smoker conductivity typically falls far from the prediction line but no clear trend emerges. A very similar result was obtained for the left calf.

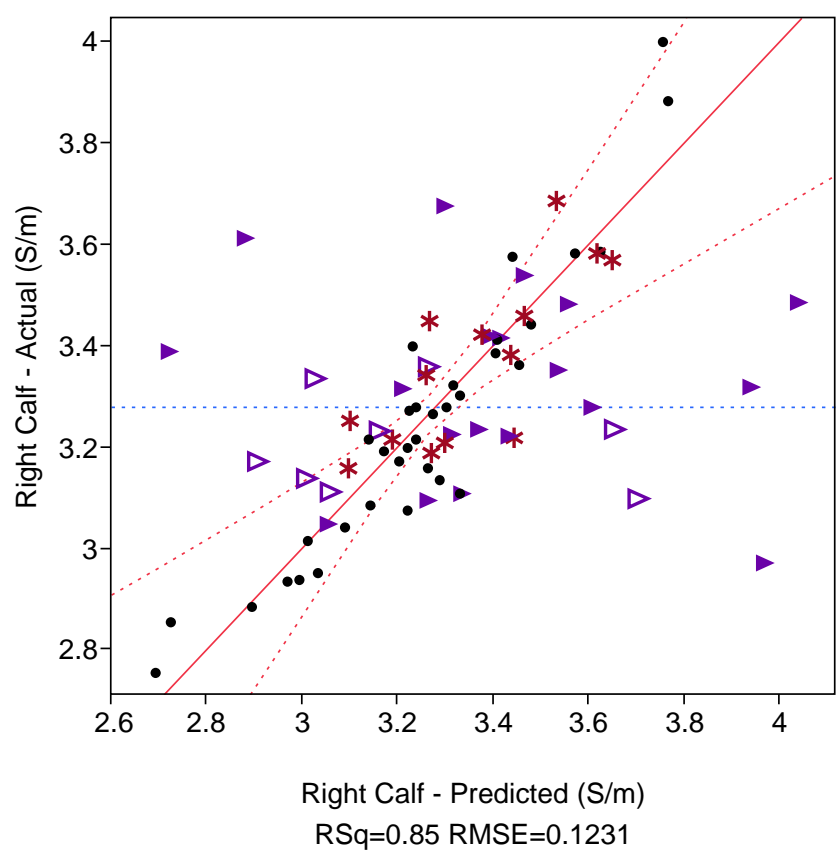

Fig.10 Overlay of smoker conductivities (triangles) onto plot of conductivity model for right calves of nonsmokers. Open triangles belong to diabetics, starred points belong to risk group one and remaining data belong to nonsmokers not in risk group one.

Our first clinical study, involving only "healthy" subjects revealed that electrical conductivity at the mid volar forearm typically increases by about $10 \%$ when elevated for 60 seconds. Figure 11 shows the result of a similar test, though duration of arm elevation time was reduced to 30 seconds, and ICCS measurement was taken at the proximal location instead. Data are sorted first by smoking (1 - 48 are nonsmokers; 4978 are smokers) and then by age (subjects of greatest age in each category appear as \#48 and \#78 on the plot).

As is evident from the overlay plot, the two changes in testing protocol likely contributed to the observed reduction in displacement compared to the previous study. Recall figure 4, and also figure 7 which shows the mid volar forearm exhibiting lower conductivities. Nevertheless, figure 4 is used as a kind of reference for figure 11 in the sense that behavior observed in figure 4 is considered to represent a normal pattern in healthy adults. That is, healthy subjects with a somewhat elevated blood pressure are expected to exhibit a smaller elevation-induced positive conductivity displacement while those with lower blood pressure should be expected to exhibit a much higher positive conductivity displacement.

Diamond shaped green-colored symbols are used to denote subjects from risk group one, while subjects with high rightarm systolic blood pressure are indicated with open symbols, regardless of risk group affiliation. Three data points, belonging to subjects in risk group one, fall in the "high blood pressure" category. Two of these fall below the mean and could be considered normal, while just one is well above the mean and fails to follow the "pattern". Thus, only 1 of 14 data points from the healthiest risk group fail to follow the pattern expected for normal or healthy individuals.

Excluding all subjects from risk group one, 16 of 64 data points come from subjects with elevated right-arm systolic blood pressure and mean displacement at or above the mean. This represents $25 \%$ of all those subjects not part of risk group one, which compares to $7 \%$ from the first risk group showing higher than expected displacement. Simple examination of figure 11 shows that nearly all unexpected positive conductivity displacements come from those subjects that are either older ( $>50$ ), or who smoke. Regardless of age, $33 \%$ of those who smoke exhibit both high blood pressure and a positive conductivity displacement at or above the mean.

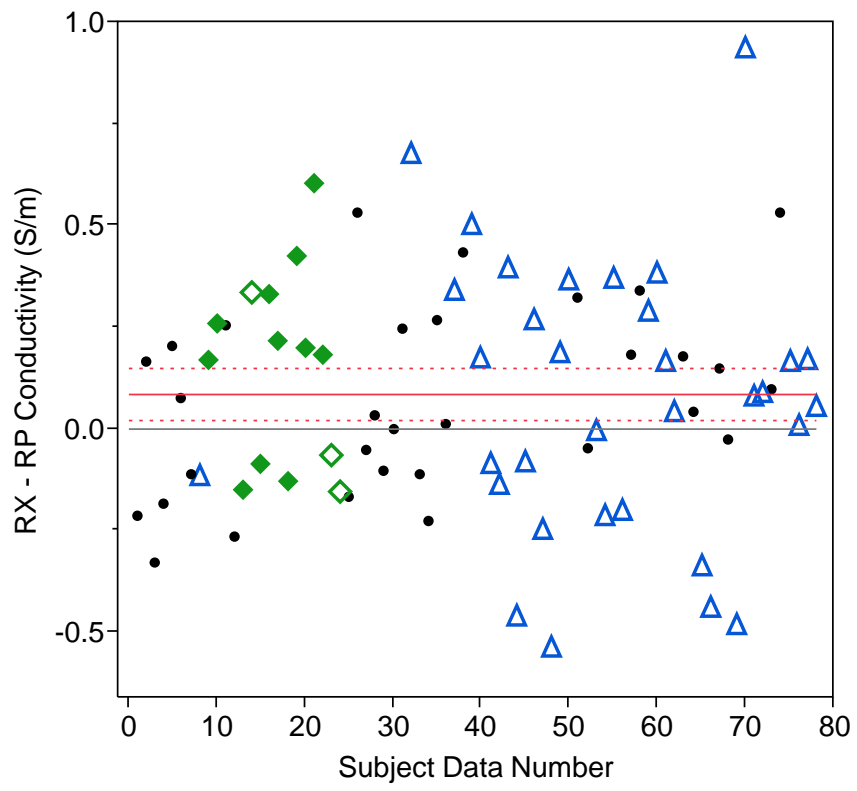

Fig.11 Paired comparison of conductivities of right proximal volar forearm, elevated and not elevated. Open symbols designate those subjects showing high right arm systolic blood pressure; diamond symbols belong to risk group 1 subjects. Data are sorted by smoking first, then by age.

Figure 11 clearly shows that negative conductivity displacements are also a feasible response to extremity elevation changes. Again this appears to be more prevalent in those who are older than 50 (beginning with data point 33 for non-smokers), or in those who regularly smoke. This also happened in the first clinical study involving only healthy 
subjects, but was rare. Here it is relatively common, with about five data points indicating a displacement of approximately $-0.5 \mathrm{~S} / \mathrm{m}$.

A plot similar to that of figure 11 was also prepared for the calf sites. Figure 12 shows the result for the left calf, clearly showing the extent of conductivity displacement to be rather substantial, averaging about $1.3 \mathrm{~S} / \mathrm{m}$ for the entire group.

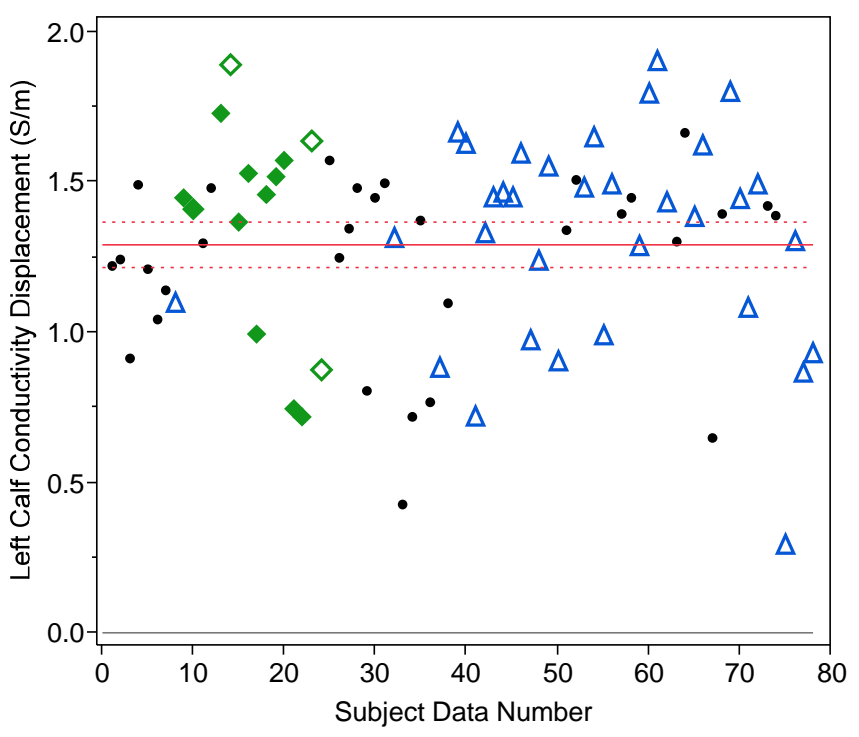

Fig.12 Paired comparison of conductivities of left calf, elevated and not elevated. Open symbols designate those subjects showing high right arm systolic blood pressure; diamond symbols belong to risk group 1 subjects. Data are sorted by smoking first, then by age.

Again, if figure 4 provides a kind of reference that reveals a pattern corresponding to normal for healthy individuals, then subjects with high blood pressure should present a conductivity displacement no higher than about the mean. Two of seven subjects in risk group one violate the normal pattern, while 13 of 30 violate the pattern among all smokers.

Referring again to figure 12, the two data points from risk group one exhibiting the smallest conductivity displacement belongs to the same subject - measured at about $0.75 \mathrm{~S} / \mathrm{m}$. These points nearly fall on top of each other and are somewhat difficult to distinguish. Of interest is the fact that this subject was taller than any other subject by about six inches. This result was sufficient cause for us to reconsider once again whether 30 seconds of extremity elevation was sufficient to produce a stable conductivity reading. Given this subject's height, it was likely that fluids redistribution was still underway when the measurement was taken 30 seconds after elevation.

Since calf elevation produced a substantial increase in electrical conductivity relative to the sitting position (with extended leg), it was expected that conductivity of the calf while standing would produce the lowest values. As figures 13 and 14 clearly reveal, this was not the case. Not only are mean conductivities at the calf much higher while in a standing position than when in the sitting position, they are distributed more broadly about their averages. In fact, the mean conductivity at the left calf while standing is nearly as high as that measured when the calf is elevated above the heart, as shown in figure 13. However, mean conductivity at the right calf, which was measured prior to the left calf, is about midway between that measured for elevated and standing positions, and more broadly distributed than that measured on the standing left calf.

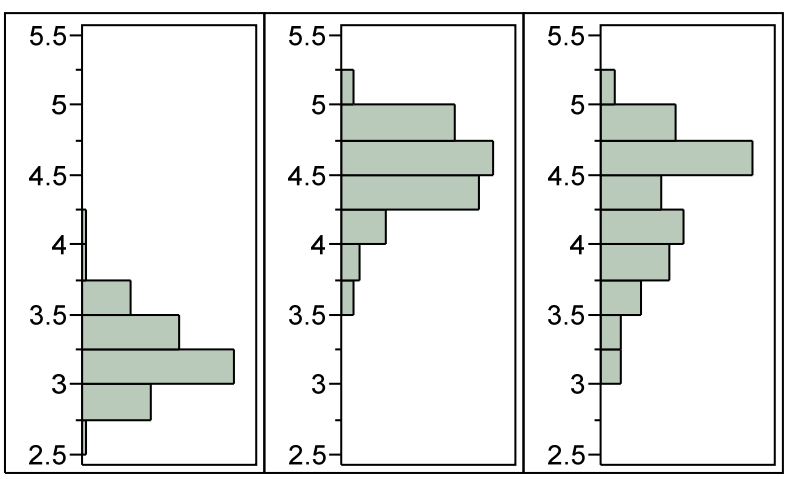

Fig.13 Left Calf Electrical Conductivity Distributions (S/m): Sitting with leg extended (3.23 \pm 0.26$)$; Elevated calf $(4.52 \pm 0.30)$; Standing (4.28 \pm 0.50$)$. Horizontal axes are number of data points in each 0.25 $\mathrm{S} / \mathrm{m}$ increment.

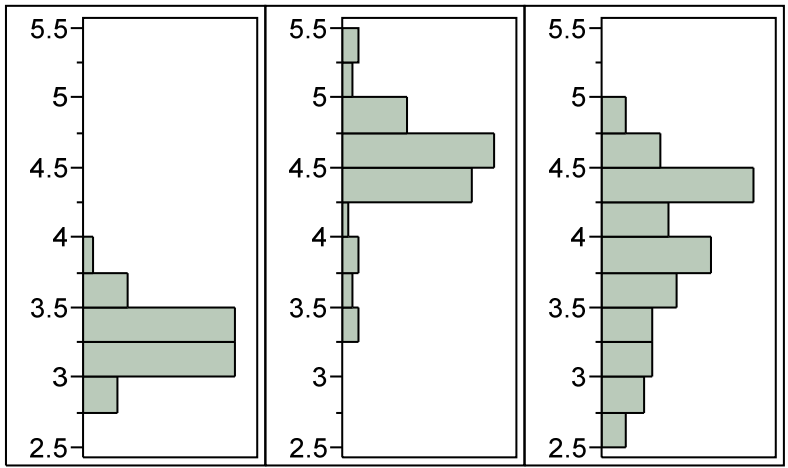

Fig.14 Right Calf Electrical Conductivity Distributions (S/m): Sitting with leg extended (3.28 \pm 0.22$)$; Elevated (4.51 \pm 0.40$)$; Standing $(3.87 \pm 0.60)$. Horizontal axes are number of data points in each $0.25 \mathrm{~S} / \mathrm{m}$ increment.

All of these effects are attributed to the compressive stresses imposed by tightened calf muscle on the vascular tree. While a subject was standing, the nurse noted that calf muscles were stiffened. If compression played a role, as we believe it did, blood would only be free to collect, or pool, in the calf region once the muscle was relaxed. Typically, calf muscle tissue had only been relaxed two to three seconds at most before conductivity measurements were taken. In 
hindsight, we now suspect that this time was far too short to allow for appreciable redistribution of blood and interstitial fluid. Since full weight was put on the left calf muscle prior to its measurement (never the case for the right calf), redistribution of fluids into the vascular tree permeating the left calf was even more impaired. If calf conductivity in the standing position is to be measured at all, a better protocol is in order.

Considering the variety of measurements taken involving elevation changes, we believe there is a characteristic drainage or relaxation time that must be exceeded if a relatively stable conductivity reading is desired. Otherwise, transient effects modify what is measured. Given the results of figures 13 and 14 , the left calf is only in the early stages of filling, while the right calf is further along, but still far from stabilized.

\section{Discussion/CONCLUSIONS}

Our purpose in conducting these two clinical studies has been to determine the extent to which a variety of factors affect the electrical conductivity of soft tissue. In addition to a number of "subject variables", we have specifically focused on considering the role of gender, age, BMI and smoking. Given that the latter three are considered to increase the risk of PVD, our second study included smokers, older subjects, and those with excessive BMI. Moreover, we also examined the effect of extremity elevation on electrical conductivity, with a special interest in the role that health factors, such as smoking, play in affecting elevation-induced conductivity displacements.

The first clinical study revealed that women have a higher electrical conductivity than men at every site, though some sites such as thigh and calf were much higher. Remarkably, a paired comparison test of male and female mean conductivities showed that values for corresponding right and left side sites fell in nearly the same location - figure 1 . This result provides convincing evidence that ICCS conductivity measurements are providing a meaningful measure of local physiology inasmuch as results for left side and right side sites really ought to be the same for healthy adults.

Higher female conductivity was shown to be the result of a higher percentage of water in men. Thus, gender could be eliminated as a variable when building models to explore possible conductivity dependence on more routinely measured parameters. Fit models revealed that percent body water played the dominant role in determining measured conductivity - figure 2 shows decreased ICCS conductivity with increased body water as measured on a Tanita scale.

Of particular interest was the discovery that conductivity declines with age at all five sites where age was found to be significant. This result is consistent with a study published by Jayasree et. al. [5], which reported an increase in vascular volume with increased age. If our hypothesis is correct that electrical conductivity provides some measure of the relative volumes of vascular and interstitial tissues, an increased vascular volume with age should lead to decreased conductivity with increased age. This is what was found. Furthermore, the results of the second clinical strongly show that electrical conductivity in the upper extremities is lower in smokers, suggesting that smoking has the same impact on the microvasculature as aging.

Data from both clinical studies convincingly demonstrate that ICCS conductivity measurements on extremities are sensitive to elevation. When a limb is elevated, blood would be motivated to exit venules more readily, but also encounter increased difficulty entering and filling capillaries via arterioles. Thus, electrical conductivity should typically increase in response to increased elevation as it promotes decreased volume fraction of the microvasculature due to blood pressure reduction. Especially convincing are the roles that both systolic and diastolic blood pressures were shown to play on the extent of conductivity displacement in response to elevation change. We suggest that the results of figure 4 provide a kind of signature of what to expect from healthy individuals and that elevation-induced conductivity changes can be used in conjunction with blood pressure measurements to reach meaningful conclusions about the state of soft tissue microvasculature.

Conductivity displacement measurements, in response to elevation changes, were commonly found to fall into abnormal ranges for those placed into higher risk groups. As demonstrated on healthy subjects in our first study, individuals with high blood pressure should show conductivity displacements beneath the mean for the subject group. Subjects from the second study with a variety of risk factors were shown to be much more likely to violate this criterion than those in the low risk group. In particular, smokers with high blood pressure were highly likely to show conductivity displacements above the group mean. We suggest that this could be the result of blockage on the arterial side of the vascular circuit, preventing development of the normal level of blood volume one would expect from a high blood pressure individual.

We now know that a decrease in conductivity is also possible in response to limb elevation, since this has been repeatedly observed at the forearm. Conductivity decrease will likely depend upon a number of factors, which could include blockages present at a variety of locations along the vascular circuit, and also the extent to which interstitial fluid itself may drain out of a measurement site via the microvasculature. Drainage of interstitial fluid may be partly encouraged simply because of the pressure exerted at the site due to placement of the ICCS unit firmly against the skin. This effect is discussed and modeled in depth by Leiderman et. al. [7], indicating a wide range of relaxation times for drainage, depending upon vessel leakage, tissue mechanical properties and loading. Considering a worst case scenario, if a subject were to have a combination of significant venous blockage, elevated blood pressure, but ample opportunity for interstitial drainage via microvasculature such as lymphatic vessels, electrical conductivity could plummet in response to 
extremity elevation. This has been observed in higher risk subjects participating in the second study.

An unexpected result in our second clinical trial was the rather high electrical conductivities observed in the feet of most subjects. During measurement, the nurse noted that the feet of most subjects felt cold when held in position for ICCS measurement using her gloved hand. We also noted that the examination room temperature was $68 \mathrm{~F}, 4 \mathrm{~F}$ cooler than during the first study. Though not planned at the outset, this glitch in our preparation for the second study led to a result that provides further support for our hypothesis, that reduced blood volume in an extremity implies lowered vascular volume fractions, and in turn a higher conductivity. A cooler environment is expected to cause some reduction in blood flow to the extremities, especially those farthest from the heart.

Ideally, electrical conductivity should be measured in an elevated limb as a function of time beginning at the instant of elevation, leading to capture of transient behavior, perhaps over an interval lasting up to two minutes or more. Tschakovsky and Hughson [8] published data suggesting that venous emptying, as measured by drop in forearm volume in response to forearm elevation, is nearly stabilized after about $30 \mathrm{~s}$. Their results were obtained from a group of healthy subjects and showed some interesting transient behavior beneath $30 \mathrm{~s}$, which we missed. Of course, the actual relaxation time will depend on a variety of factors, including temperature in the extremity and degree of muscle relaxation as well as health factors. Given that higher blood pressures tend to maintain blood volume and that pressures in our second panel run considerably higher, 30 seconds may be too short to achieve a stable result - only one of our subjects from the second study had a right arm diastolic pressure beneath 65 $\mathrm{mm} \mathrm{Hg}$. In the case of our measurements at the calf while in the standing position, the problem of insufficient "settling" time is pronounced, since calf muscles had been relaxed for no more than about three seconds. In addition to the relaxation effects resulting from elevation changes and muscle tightness, there are also the relaxation processes associated with contact pressure developed between the ICCS unit and the skin. As already mentioned, application of surface loads can cause interstitial fluid drainage via the microvasculature. "Drainage" times might be as high as 300 seconds for normal tissue, though for tumors, much smaller [7].

Though the current ICCS unit has performed quite well in two different clinical trials, there were a few issues noted during use, which could result in substantial improvement in measurement if fully addressed. One concerns the tendency for the instrument to drift about 3.5\% over about two hours of continuous operation. Evaluation of a subject during clinical testing lasted about 12 minutes, so the extent of drift was much less, about $1.6 \%$. Nevertheless, the desire to do longer term testing, where physiological changes may be subtle over time, requires that drift be eliminated. Also, noise was considerable, though this was addressed reasonably well with signal averaging. But if short term transients are desired, then noise must also be greatly reduced.

Another measurement issue concerns the use of the ICCS instrument as a hand-held unit. During use, holding the ICCS instrument precisely in the desired location may be cumbersome. Placement is especially an issue if a particular site is abandoned for a period of time and then returned to returning to exactly the same site as before may be an issue. In our testing on numerous subjects, we found that conductivity data at mid volar and proximal volar forearm locations were highly correlated, suggesting that precise return to a site is not critical. In spite of that, accurate determination of conductivity changes over time dictates a preferred strategy where the ICCS unit is maintained at the measurement location for the time period of interest. To that end, our plan is to move to a compact, strap-on module that can be kept at the desired location for extended periods of time, ranging from a few minutes to a few hours. For the longer time periods, the module must keep the induction coil in contact with the skin but not apply so much pressure that the soft tissue at the site is itself significantly disturbed by the unit.

\section{REFERENCES}

[1] Heller, J., Feldkamp, J.R. (2009). Auto-tuned induction coil conductivity sensor for in-vivo human tissue measurements. Meas. Sci. Rev., 9, No. 6, 162-168.

[2] Holder, D.S. (ed.) (2004). Electrical Impedance Tomography: Methods, History and Applications. Institute of Physics.

[3] Haemmerich, D., Staelin, S.T., Tsai, J.Z. (2003). In vivo electrical conductivity of hepatic tumors. Physiol. Meas. , 24, 251-260.

[4] Stinstra, J.G., Shome, S., Hopenfeld, B., Macleod, R.S. (2005). Modeling the passive cardiac electrical conductivity during ischemia. Med. Biol. Comput. Nov., 43 (6), 776-782.

[5] Jayasree, V.K., Sandhya, T.V., Randhakrishnan, P. (2008). Non-invasive studies on age-related parameters using a blood volume pulse sensor. Meas. Sci. Rev., 8, (4), 82-86.

[6] Frank, S.M, Norris, E.J., Christopherson, R., Beattie, C. (1991). Right- and left-arm blood pressure discrepancies in vascular surgery patients. Anesthesiology, 75, 457-463.

[7] Leiderman, R., Barbone, P.E., Oberai, A.A., Bamber, J.C. (2006). Coupling between elastic strain and interstitial fluid flow: ramifications for poroelastic imaging. Phys. Med. Biol., 51, 6291-6313.

[8] Tschakovsky, M.E., Hughson, R.L. (2000). Venous emptying mediates a transient vasodilation in the human forearm. Am. J. Physiol.: Heart Circ. Physiol., 279, H1007-H1014. 